\title{
Collective Thomson scattering data analysis for Wendelstein 7-X
}

Abramovic, I.; Pavone, A.; Svensson, J.; Moseev, D.; Salewski, Mirko; Laqua, H.P.; Cardozo, N.J. Lopes; Wolf, R.C.

Published in:

Journal of Instrumentation

Link to article, DOI:

10.1088/1748-0221/12/08/C08015

Publication date:

2017

Document Version

Peer reviewed version

Link back to DTU Orbit

Citation (APA):

Abramovic, I., Pavone, A., Svensson, J., Moseev, D., Salewski, M., Laqua, H. P., Cardozo, N. J. L., \& Wolf, R. C. (2017). Collective Thomson scattering data analysis for Wendelstein 7-X. Journal of Instrumentation, 12(08), [C08015]. https://doi.org/10.1088/1748-0221/12/08/C08015

\section{General rights}

Copyright and moral rights for the publications made accessible in the public portal are retained by the authors and/or other copyright owners and it is a condition of accessing publications that users recognise and abide by the legal requirements associated with these rights.

- Users may download and print one copy of any publication from the public portal for the purpose of private study or research.

- You may not further distribute the material or use it for any profit-making activity or commercial gain

- You may freely distribute the URL identifying the publication in the public portal

If you believe that this document breaches copyright please contact us providing details, and we will remove access to the work immediately and investigate your claim 


\title{
Collective Thomson scattering data analysis for Wendelstein 7-X
}

\author{
I. Abramovic ${ }^{a, b, 1}$ A. Pavone,${ }^{b}$ J. Svensson,${ }^{b}$ D. Moseev, ${ }^{b}$ M. Salewski ${ }^{c}$ H. P. Laqua,${ }^{b}$ \\ N. J. Lopes Cardozo,${ }^{a}$ R. C. Wolf ${ }^{b}$ \\ ${ }^{a}$ University of Technology Eindhoven, De Zaale, 5612 AJ Eindhoven, The Netherlands \\ ${ }^{b}$ Max - Planck Institute fur Plasma Physik, Wendelsteinstraße 1, D-17491 Greifswald, Germany \\ ${ }^{c}$ Technical University of Denmark, Fysikvej, 2800 Kgs. Lyngby, Denmark \\ E-mail: i.abramovic@tue.nl
}

Abstract: Collective Thomson scattering (CTS) diagnostic is being installed on the Wendelstein 7-X stellarator to measure the bulk ion temperature in the upcoming experimental campaign. In order to prepare for the data analysis, a forward model of the diagnostic (eCTS) has been developed and integrated into the Bayesian data analysis framework Minerva. Synthetic spectra have been calculated with the forward model and inverted using Minerva in order to demonstrate the feasibility to measure the ion temperature in the presence of nuisance parameters that also influence CTS spectra. In this paper we report on the results of this anlysis and discuss the main sources of uncertainty in the CTS data analysis.

KEYwORDS: nuclear instruments and methods for hot plasma diagnostics, analysis and statistical methods, simulation methods and programs

\footnotetext{
${ }^{1}$ Corresponding author.
} 


\section{Contents}

1 Introduction 1

2 CTS on Wendelstein 7 - X 2

3 Analysis of collective Thomson scattering data 2

3.1 Forward modelling 2

3.2 Bayesian inversion 3

4 Results $\quad 3$

4.1 Sensitivity to the plasma parameters and the scattering geometry 3

4.2 Bayesian inference from synthetic spectra 4

5 Discussion 5

6 Conclusions 6

\section{Introduction}

A Collective Thomson Scattering (CTS) diagnostic is being developed for the Wendelstein 7-X stellarator. The diagnostic allows the measurement of the ion temperature, isotope ratio, fast ion velocity distribution function, plasma rotation, and MHD activity. The diagnostic principle is based on the microwave scattering off the collective fluctuations in the plasma. The scattering is collective if the condition $\alpha \geq 1$ is satisfied, where $\alpha$ stands for the Salpeter parameter defined as $\alpha=\frac{1}{\lambda_{d} k^{\delta}}$, where $k^{\delta}=\left|\vec{k}_{s}-\vec{k}_{i}\right|$ and $\lambda_{d}$ is the Debye length. The wave vectors $\vec{k}_{i}$ and $\vec{k}_{s}$ correspond to the incident and the scattered wave, respectively. This condition translates into constraints on the probing frequency and the scattering geometry. The probing beam is scattered off the fluctuations in the electron density, the current density, the electric field, and the magnetic field. The fluctuations are resolved along the direction $k^{\delta}$. The diagnostics of this type are installed and successfully exploited at other fusion experiments such as ASDEX Upgrade [1], LHD [2], and TEXTOR [3]. CTS will also be installed in ITER for alpha particle measurements [4].

The CTS measurements result in scattering spectra. The shape and width of the spectra are influenced by a variety of plasma parameters. Therefore forward modelling in conjunction with the Bayesian formalism is typically employed for the inference of parameter values from the spectra [1]. The forward model has to account for the plasma response to the probing beam, as well as for the coupling of the probing beam to the collective fluctuations which gives rise to the scattered field. Hence we can gain insight by studying the sensitivity of the forward model to the plasma parameters and the scattering geometry. The sensitivity analysis entails the calculation of synthetic spectra which can be inverted through the Bayesian framework. The purpose of this is to show that 
the correct parameter values can be recovered from synthetic spectra by Bayesian inference.

In this paper we report on the preparation for the data analysis for the first CTS measurements on Wendelstein 7-X: the sensitivity of the developed forward model (eCTS) and the Bayesian inference on plasma parameters and scattering geometry. The Bayesian inference is done within Minerva - the data analysis framework used on Wendelstein 7-X. The two configurations available for the CTS diagnostic are outlined in Section 2. The method which will be used for the data analysis is described in Section 3. In Section 4 we present the results of the sensitivity analysis of the forward model and the Bayesian inversion of the synthetic spectra. The discussion of the results and the conclusion are given in Sections 5 and 6, respectively.

\section{CTS on Wendelstein 7 - X}

The CTS diagnostic on Wendelstein 7-X will use one of the $140 \mathrm{GHz}$ gyrotrons for electron cyclotron resonance heating as the source of the probing beam. The measurements will be done at two different locations in the plasma (see Figure 1). The scattering geometry will be more flexible in the bean shaped cross section (Figure 1 right) allowing the observation angle, $\phi=\angle\left(\vec{B}, \vec{k}^{\delta}\right)$, in the range of $75-115$. Measurements in the center of the plasma will not be possible in this cross section (Figure 1 right) due to the absorption of the beam in the center of the plasma [5]. In the triangular cross section the observation angle in the center will be $\phi=110$ (Figure $1 \mathrm{left}$ ) [5]. Steering of the probing radiation from one location to the other will be done using a remote steering launcher. The spectral range covered by the receiver is $138-142 \mathrm{GHz}$. The choice of the polarization of the probing and received radiation depends on the presence of cut-offs and the spectral power density of the electron cyclotron emission reaching the receiver. In Figure 1 the probing and receiver beams are indicated. The red colour indicates the absorption of the probing radiation. The overlap volume, from which the CTS signal originates, is indicated by a blue circle at the intersection of the probing and receiver beams. The position of the overlap volume is a source of uncertainty in the CTS measurements and has to be accounted for by ray tracing after each shot.

\section{Analysis of collective Thomson scattering data}

\subsection{Forward modelling}

The forward model eCTS calculates the spectral power density in the observable spectral range. The spectral power density is given by [6]:

$$
\frac{\partial P^{s}}{\partial \omega}=P^{i} O_{b} \frac{\omega^{i} \omega^{s}}{2 \pi c^{2}} r_{e}^{2} n_{e} G S\left(\vec{k}^{\delta}, \omega\right)
$$

where $r_{e}$ is the classical electron radius, $c$ is the speed of light in vacuum, $n_{e}$ is the electron density, $O_{b}$ is the beam overlap, $G$ is the geometrical form factor, $S\left(\vec{k}^{\delta}, \omega\right)$ is the spectral density function, and $\omega=\omega^{s}-\omega^{i}$. The geometrical form factor, $G$, is calculated following the approach in [7]. It accounts for the polarizations of the probing and scattered radiation. The spectral density function, $S\left(\vec{k}^{\delta}, \omega\right)$, is calculated in the electrostatic approximation rather than with a full electromagnetic treatment. To produce the synthetic spectra the forward model takes the following parameters as input: incident radiation mode, scattered radiation mode, incident power $P_{i}$, probing frequency $v^{i}$, 


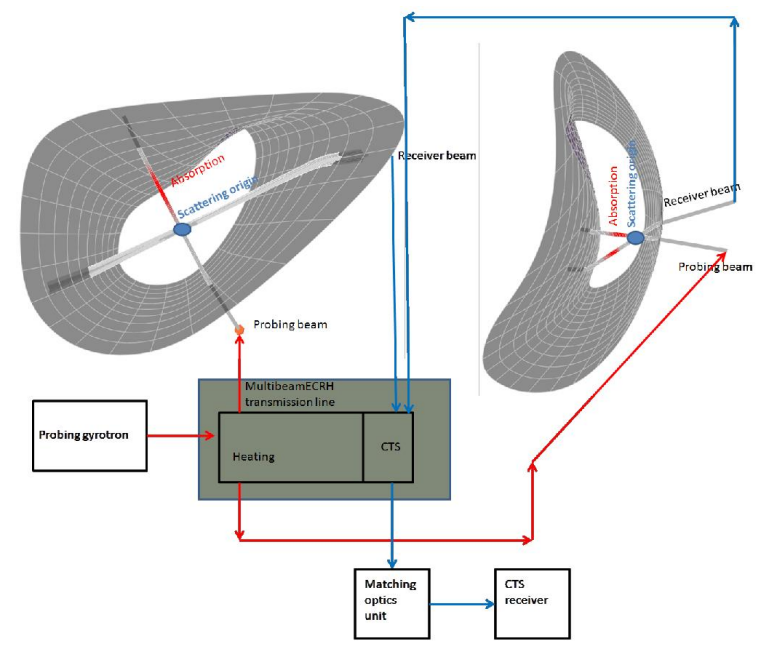

Figure 1. Block scheme of CTS on Wendelstein 7-X [5]. Showing two possible measurements locations:in the triangular cross section (left) and in the bean cross section (right). Probing and receiver beams are indicated for each cross section. The scattering volume (from which the CTS signal originates) is indicated by the blues circle at the intersection of the beams. The red colouring indicates the absorption of the probing radiation.

overlap volume $O_{b}$, electron density $n_{e}$, ion density $n_{i}$, electron temperature $T_{e}$, ion temperature $T_{i}$, strength of the magnetic field $B$, projection angle $\phi=\angle\left(\vec{B}, \vec{k}^{\delta}\right)$, and the angle between the probing and scattered waves $\theta=\angle\left(\vec{k}^{i}, \vec{k}^{s}\right)$.

\subsection{Bayesian inversion}

The forward model alone is not enough for the data analysis process. In order to infer the parameter values from the measured spectra, we solve the inverse problem by performing Bayesian inversion. For this purpose the eCTS forward model has been integrated into Minerva which is a completely Bayesian data analysis framework used on Wendelstein 7-X. The input parameters of the forward model are redefined within Minerva in a way which allows putting a prior probability distribution on each parameter. Forward models of different diagnostics in Minerva can be interconnected through defined dependencies. This block structure allows decoupling between the data sources, forward models of the diagnostics, and the physics models implemented in the framework [8]. The inversion is done by the MAP algorithm which maximizes the posterior distribution of the free parameters given the data [8].

\section{Results}

\subsection{Sensitivity to the plasma parameters and the scattering geometry}

Sensitivity of the forward model to a selection of nuisance parameters is investigated. Synthetic spectra have been calculated varying one input parameter at a time while keeping all others fixed. The default configuration which was varied had the following values of nuisance parameters: electron density $n_{e}=6 \times 10^{19} \mathrm{~m}^{-3}$, ion density $n_{i}=6 \times 10^{19} \mathrm{~m}^{-3}$, electron temperature $T_{e}=3 \mathrm{keV}$, ion temperature $T_{i}=3 \mathrm{keV}$, projection angle $\phi=110^{\circ}$, and the angle between the probing and 
scattered waves $\theta=100^{\circ}$. In Figure 2 the results are shown for varying the ion density (left), $n_{i}$ , and ion temperature $T_{i}$ (right) for a hydrogen plasma. Figure 3 shows the sensitivity of the
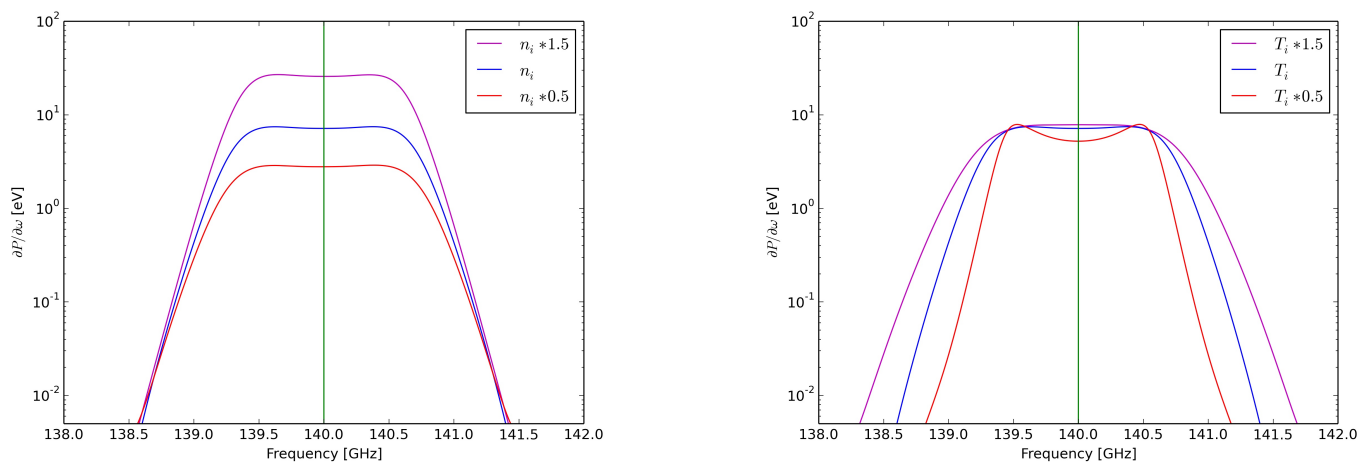

Figure 2. Sensitivity of the spectra to the changes in the ion density (left), $n_{i}$, and the ion temperature (right) $T_{i}$. The parameter indicated in the legend has been varied while the others were kept fixed.
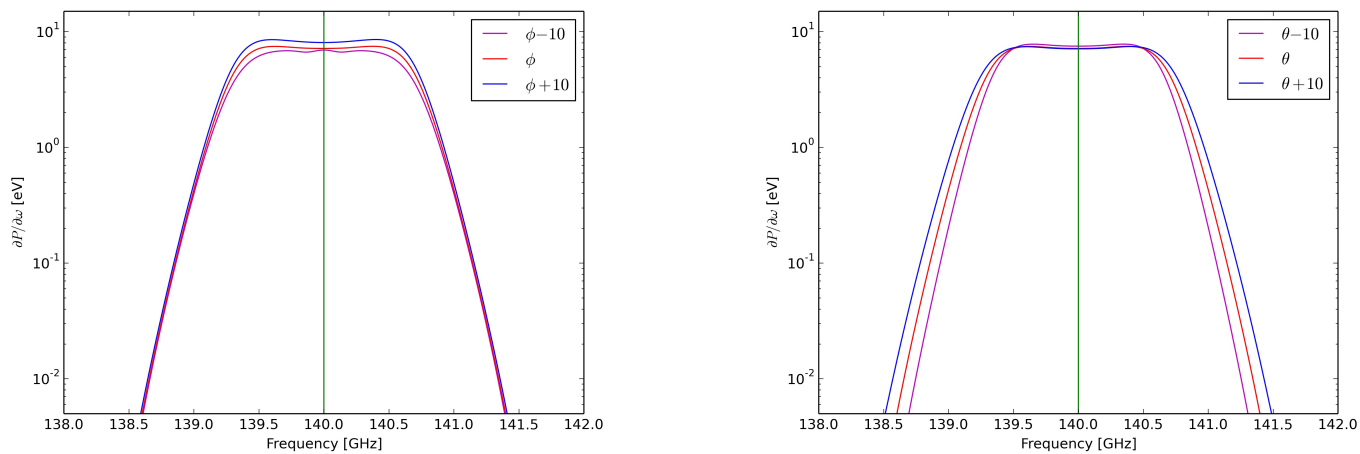

Figure 3. Sensitivity of the spectra to the changes in angles characterizing the scattering geometry: $\phi=$ $\angle\left(\vec{B}, \vec{k}^{\delta}\right)$ (left), and $\theta=\angle\left(\vec{k}^{i}, \vec{k}^{s}\right)$ (right). The parameter indicated in the legend has been varied while the others were kept fixed.

forward model to the two angles characterizing the scattering geometry. Notice that the width of the spectrum is sensitive to the change in the angle $\theta$ (Figure 3 right) and the change in ion temperature $T_{i}$ (Figure 2 right).

\subsection{Bayesian inference from synthetic spectra}

The synthetic spectra produced by the forward model have been fed into the Minerva framework as observed data. The parameter which was varied in the sensitivity analysis (see previous section 4.1) has been assigned a normal prior probability distribution. The results of the Bayesian inference of the ion temperature, $T_{i}$, and the scattering angle, $\theta$, are given in Figure 4. Since no noise has been added to the synthetic spectra, and the only parameter with a prior distribution is the one whose value is inferred, the inversion results in spectra which overlap with the original synthetic spectra. 

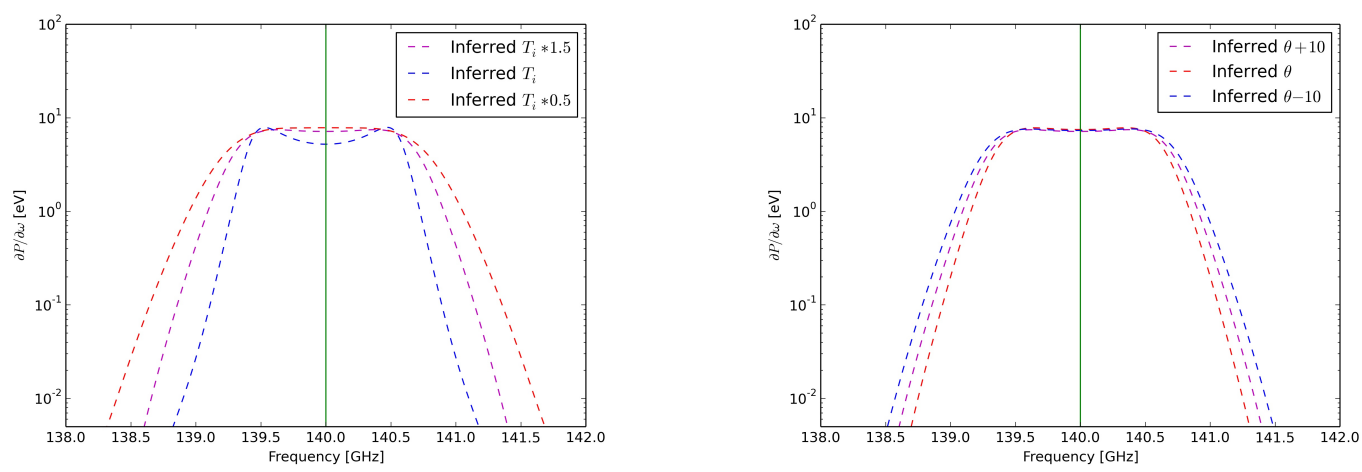

Figure 4. Spectra obtained by inversion of the synthetic spectra given in Figures 2 and 3 for varying $T_{i}$ (left), and $\theta$ (right).

The input and inferred values of the ion temperature, $T_{i}$, and the scattering angle, $\theta$, are given in Table 1.

Table 1. The ion temperatures and scattering angles inferred from the synthetic data.

\begin{tabular}{|l|l|l|l|}
\hline Input $T_{i}[\mathrm{keV}]$ & Inferred $T_{i}[\mathrm{keV}]$ & Input $\theta\left[^{\circ}\right]$ & Inferred $\theta\left[^{\circ}\right]$ \\
\hline 1.5 & 1.49 & 90 & 91.57 \\
3 & 2.99 & 100 & 100.34 \\
4.5 & 4.49 & 110 & 110.09 \\
\hline
\end{tabular}

\section{Discussion}

The results obtained in Section 4 demonstrate that the width of the CTS spectra is affected by the changes in the ion temperature $T_{i}$, and the scattering angle $\theta$ (see Figure 3 right). It is therefore necessary to obtain the values of the scattering angles from ray tracing prior to Bayesian inference in order to minimize the uncertainty on the inferred value of the ion temperature. Notice that a change in the angle $\theta$ results in a change in the length of the scattering wave vector $\vec{k}^{\delta}$. This is directly related to the position of the overlap volume. Another source of uncertainty in the CTS data analysis is the size of the overlap volume. The finite size of the overlap volume is not taken into account in the forward model. It is implicit that the error introduced by assuming one $\vec{k}^{\delta}$ is small. In Section 4.2 we have inferred the correct values of the ion temperature, $T_{i}$, and the scattering angle, $\theta$, by performing Bayesian inversion of the synthetic spectra from Section 4.1. However, inference from real measurements will be more challenging due to the high noise levels and uncertainties in several parameters necessary for the calculation of the spectral power density (see equation 3.1). Within Minerva we can obtain the values of other parameters by linking the corresponding nodes of our model to the data signals of diagnostics measuring the necessary parameters. A typical example is the electron temperature, $T_{e}$, from electron cyclotron emission diagnostic or incoherent Thomson scattering measurements. In addition we can introduce a scaling factor as a free node in the model accounting for the uncertainty in the absolute scaling according to equation 3.1. 
Our forward model has been developed based on the electrostatic approximation [7]. The applicability of this approximation is limited by the coupling of the longitudinal and the transverse modes in a magnetized plasma [9]. The electromagnetic effects in the bulk ion region have not been fully investigated. Comparison of our electrostatic forward model with a fully electromagnetic model within Minerva can lead to a better understanding of the limitations of the electrostatic model in this region.

\section{Conclusions}

Forward modelling and synthetic spectra analysis have been done in preparation for the analysis of real data from the CTS diagnostic on Wendelstein 7-X. We have demonstrated the sensitivity of the synthetic CTS spectra to the changes in the ion density, ion temperature and the scattering angles. The sensitivity of the synthetic spectra to the changes in a selection of nuisance parameters is in agreement with the theoretical predictions [1].

\section{References}

[1] M. Salewski et al., Comparison of fast ion collective Thomson scattering measurements at ASDEX Upgrade with numerical simulations, Nuclear Fusion vol. 50, (2010).

[2] M. Nishiura et al., Spectrum response and analysis of $77 \mathrm{GHz}$ band collective Thomson scattering diagnostic for bulk and fast ions in LHD plasmas, Nuclear Fusion vol. 54, (2014).

[3] D. Moseev et al., Comparison of measured and simulated fast ion velocity distributions in the TEXTOR tokamak, Plasma Physics and Controlled Fusion, vol. 53, (2011).

[4] M. Salewski et al., Impact of ICRH on the measurement of fusion alphas by collective Thomson scattering in ITER, Nuclear Fusion vol. 49, (2009).

[5] D. Moseev et al., Application of the ECRH radiation for plasma diagnostics in Wendelstein 7 X, 26 th IAEA Fusion Energy Conference, IAEA (2016).

[6] H. Bindslev, On the Theory of Thomson Scattering and Reflectometry in a Relativistic Magnetized Plasma, Risø National Laboratory (Denmark), (1992).

[7] T. Hughes, S. Smith, Effects of plasma dielectric properties on Thomson scattering of millimetre waves in tokamak plasmas, Journal of Plasma Physics vol. 42, (1989).

[8] J. Svensson, A. Werner, Large Scale Bayesian Data Analysis for Nuclear Fusion Experiments, IEEE International Symposium on Intelligent Signal Processing (2007) pg. 1-6

[9] J. D. Callen, G. E. Guest, Electromagnetic effects on electrostatic modes in a magnetized plasma, Nuclear Fusion 13. doi:10.1088/0029-5515/13/1/011

[10] T. Hughes, S. Smith, Calculations of Thomson scattering functions for alpha particle diagnostics in JET plasmas, Nuclear Fusion vol. 28, (1988). 